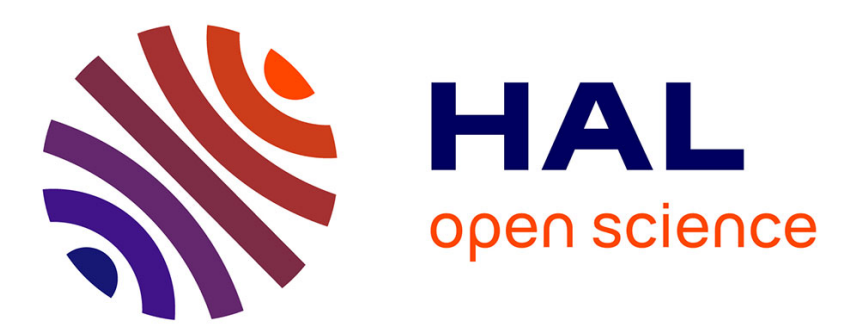

\title{
Détermination des paramètres de distribution des auto-interstitiels silicium en vue de la modélisation 2-D des processus technologiques. Discussion sur la validité physique \\ E. Scheid, P. Chenevier
}

\section{To cite this version:}

E. Scheid, P. Chenevier. Détermination des paramètres de distribution des auto-interstitiels silicium en vue de la modélisation 2-D des processus technologiques. Discussion sur la validité physique. Revue de Physique Appliquée, 1985, 20 (7), pp.483-491. 10.1051/rphysap:01985002007048300 . jpa-00245361

\author{
HAL Id: jpa-00245361 \\ https://hal.science/jpa-00245361
}

Submitted on 1 Jan 1985

HAL is a multi-disciplinary open access archive for the deposit and dissemination of scientific research documents, whether they are published or not. The documents may come from teaching and research institutions in France or abroad, or from public or private research centers.
L'archive ouverte pluridisciplinaire HAL, est destinée au dépôt et à la diffusion de documents scientifiques de niveau recherche, publiés ou non, émanant des établissements d'enseignement et de recherche français ou étrangers, des laboratoires publics ou privés. 
Classification

Physics Abstracts

$66.30 \mathrm{D}-66.30 \mathrm{~J}-66.30 \mathrm{~L}-81.60$

\title{
Détermination des paramètres de distribution des auto-interstitiels silicium en vue de la modélisation 2-D des processus technologiques. Discussion sur la validité physique
}

\author{
E. Scheid et P. Chenevier \\ Laboratoire de Physique des Composants à Semiconducteurs, UA-CNRS 840, ENSERG, 23, avenue des Martyrs, \\ 38031 Grenoble Cedex, France
}

(Reçu le 17 décembre 1984, révisé les 27 février et 26 mars 1985, accepté le 9 avril 1985)

\begin{abstract}
Résumé. - Nous présentons une simulation de la distribution en volume des auto-interstitiels silicium pendant une oxydation thermique en face arrière de plaquette. Nous montrons d'abord qu'elle peut permettre la description de la distribution d'impuretés à 2 dimensions dans une structure "LOCOS ». Nous donnons également les hypothèses physiques qui sont à la base de notre modèle. Ce dernier donne une longueur de recombinaison en volume, $L_{\mathrm{v}}=$ $35 \mu \mathrm{m} \pm 8 \mu \mathrm{m}$ et une longueur de recombinaison en surface sur $\mathrm{SiQ}_{2}$ de $8 \mu \mathrm{m} \pm 2 \mu \mathrm{m}\left(\mathrm{à}_{1} 100^{\circ} \mathrm{C}\right)$ qui sont en bon accord avec les ordres de grandeurs déterminés sur des mesures à 2 dimensions. La valeur trop faible trouvée pour la diffusivité $D_{\mathrm{i}}$ des auto-interstitiels et les contradictions soulevées par certains auteurs nous amènent ensuite à remettre en cause certaines hypothèses jusqu'à présent admises à propos de l'effet de l'oxydation thermique sur le silicium.
\end{abstract}

\begin{abstract}
A simulation of the distribution in volume of the silicon self-interstitials during a thermal oxidation on the backside of a wafer is presented. First, we show that it allows the description of the 2-D impurity distribution in a «LOCOS " structure. The physical assumptions of the model foundations are also given. Our model gives a volume recombination length, $L_{\mathrm{v}}=35 \mu \mathrm{m} \pm 8 \mu \mathrm{m}$ and a surface recombination length on $\mathrm{SiO}_{2}, L_{\mathrm{s}}=8 \mu \mathrm{m} \pm$ $2 \mu \mathrm{m}\left(\right.$ at $\left.1100^{\circ} \mathrm{C}\right)$ that are in good agreement with the orders of magnitude deduced from some measurements in 2 dimensions. The fact that the value for the diffusivity $D_{i}$ of the self-interstitials was found to be too weak and that there exists contradictions evocated by some authors leads us to reconsider some hypotheses until now advanced about the effect of the thermal oxidation on silicon.
\end{abstract}

\section{Introduction.}

L'évolution de la technologie des circuits intégrés vers les dimensions microniques et submicroniques a rendu nécessaire depuis quelques années l'utilisation de l'analyse bidimensionnelle des processus technologiques (programmes de simulation à 2 dimensions tels que SUPRA [1], OSIRIS [2], MOBIDIC [3]). Le chapitre de l'oxydation thermique du silicium est une partie importante de cette analyse, et dans un dispositif à 2 dimensions comprenant une zone oxydée et une zone passivée (voir Fig. 1) le problème de la redistribution des impuretés dans le silicium sous ces 2 régions reste posé. En effet, il est visible sur la figure 1 que la région de transition nécessite une description et donc une modélisation bidimensionnelle, modélisation qui est encore à l'étude. Actuellement, il est admis que la croissance d'oxyde crée des auto-interstitiels en excès qui modifient la diffusivité des impuretés (Oxidation

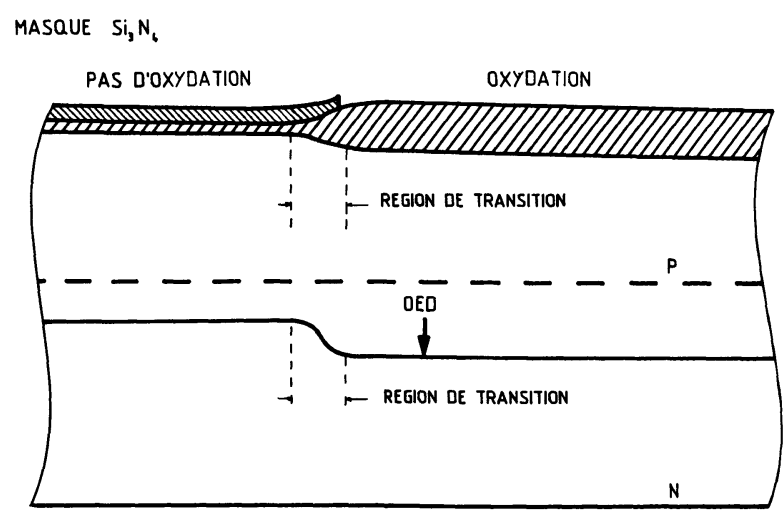

Fig. 1. - Exemple de motif étudié, nous nous intéressons surtout aux zones de transitions.

[Example of studied pattern, we are particularly interested by the transition zones.] 
Enhanced Diffusion, Oxidation Retarded Diffusion). Dans le motif déjà décrit il faut donc estimer la distribution en chaque point des auto-interstitiels (et aussi des lacunes) [4]. Cette description est rendue difficile par le fait que l'on connaît encore mal la croissance de l'oxyde dans la zone de transition (zone du bec d'oiseau) et par le manque de données sur les profils d'impuretés 2-D concernant cette géométrie. Il est considéré que la répartition des auto-interstitiels est gouvernée par leur création sous l'oxyde en croissance et leur recombinaison dans le volume du silicium et sur la surface de $\mathrm{SiO}_{2}$ passivée. Nous déterminons ces différents taux de création et de recombinaison, grâce à des simulations des expériences effectuées par Mizuo et Higuchi [5] : ceux-ci ont étudié l'influence d'une oxydation face arrière sur la redistribution d'une couche d'antimoine (entre autres impuretés) située sur la face avant; ces expériences ayant l'avantage de ne pas porter sur des zones de transitions (expériences à une dimension). A la lumière de résultats indépendants relatifs à l'existence des auto-interstitiels, nous discutons de la pertinence des hypothèses physiques sous-jacentes à ces modèles.

\section{Expériences simulées.}

Nous avons tenté de simuler les expériences faites par Mizuo et Higuchi [5] qui ont étudié la redistribution d'une couche d'impureté (ici l'antimoine) située sur la face avant d'une plaquette sous les conditions suivantes :

La face arrière est amincie localement par attaque chimique et ensuite oxydée $\left(\mathrm{O}_{2} \sec\right.$ à $\left.1100^{\circ} \mathrm{C}\right)$ au même endroit. Le reste de la face arrière est passivée par une couche $\mathrm{SiO}_{2}+\mathrm{Si}_{3} \mathrm{~N}_{4}$. Le substrat de la plaquette est soit un substrat "Czochralsky " $(\mathrm{CZ})$, soit un substrat "Floating-Zone » (FZ). L'impureté en face avant est sous une couche passivée : soit $\mathrm{SiO}_{2}+$ $\mathrm{Si}_{3} \mathrm{~N}_{4}$, soit $\mathrm{Si}_{3} \mathrm{~N}_{4}$ seul. L'épaisseur du substrat restant après amincissement va de $100 \mu \mathrm{m}$ à $400 \mu \mathrm{m}$ et le temps d'oxydation va de $500 \mathrm{~min}$ à $4000 \mathrm{~min}$ (voir Fig. 2).

Les auteurs ont constaté que l'effet de l'oxydation sur l'antimoine (ORD) ne se retrouvait de façon très nette que dans le cas substrat $\mathrm{FZ}+\mathrm{Si}_{3} \mathrm{~N}_{4}$ seul en face avant, l'influence de l'oxydation étant très atténuée dans les autres cas. Dans le premier cas, on suppose que les auto-interstitiels créés par l'oxydation diffusent à travers la plaquette et se retrouvent en face avant sans recombinaison, ni en volume (sauf avec les lacunes, voir le chapitre suivant), ni en surface, et dans les autres cas, on suppose, comme Shin et Kim [4], que les autointerstitiels se recombinent en volume dans le silicium $\mathrm{CZ}$ et/ou en surface sur le $\mathrm{SiO}_{2}$ passivé, phénomènes déjà constatés dans d'autres expériences $[6,7]$.

Une autre expérience d'oxydation face arrière a été effectuée par Taniguchi et al. [8], qu'ils ont eux-mêmes simulée. Nous verrons plus loin pourquoi nous ne nous sommes pas appuyés sur les résultats de cette expérience.

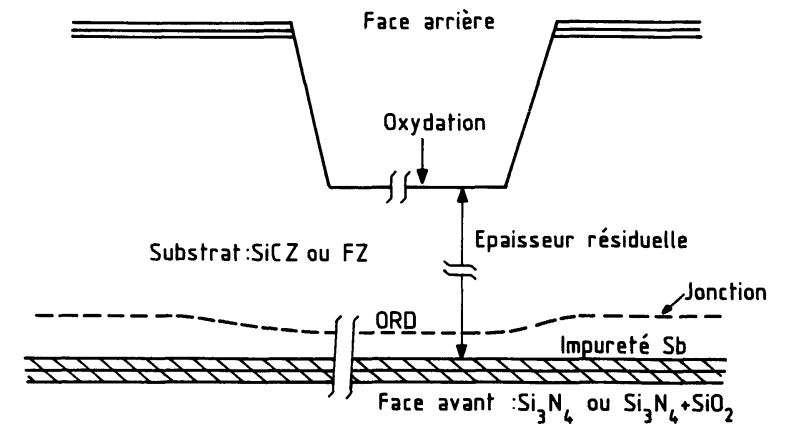

Fig. 2. - Description de l'expérience de Mizuo et Higuchi [5].

[Description of the experience of Mizuo and Higuchi [5]].

\section{Hypothèses et équations admises pour la simulation.}

3.1 TAUX DE CRÉATION DES AUTO-INTERSTITIELS. Historiquement, la sursaturation d'auto-interstitiels a été déduite de la présence et de la croissance de fautes d'empilements extrinsèques de type interstitiel, en parallèle avec une diffusivité plus grande du bore et du phosphore sous oxydation (OED) ([9] et Réfs. à l'intérieur). Ensuite, pour expliquer la diffusivité moindre de l'antimoine dans les mêmes conditions, on a supposé que les lacunes étaient dans le même temps en sous-saturation $[10,11]$. Mais alors, suivant en cela Tan et Goesele [12], la variation de la dimension caractéristique d'une faute d'empilement s'écrit :

$$
\frac{\mathrm{d} R}{\mathrm{~d} t}=\alpha\left[D_{\mathrm{i}}\left(C_{\mathrm{i}}-C_{\mathrm{isf}}\right)+D_{\mathrm{v}}\left(C_{\mathrm{vsf}}-C_{\mathrm{v}}\right)\right]
$$

où $D_{\mathrm{i}, \mathrm{v}}, C_{\mathrm{i}, \mathrm{v}}$ sont les diffusivités et concentrations des auto-interstitiels I, des lacunes $\mathrm{V}$ pendant l'oxydation et $C_{\mathrm{vsf}}, C_{\text {isf }}$ sont les concentrations d'équilibre en I et en $\mathbf{V}$ avec la faute d'empilement.

De plus, $\mathrm{d} R / \mathrm{d} t$ est reliée à la vitesse d'oxydation $\mathrm{d} E_{\mathrm{ox}} / \mathrm{d} t$ par la loi empirique ([13] et Réfs. à l'intérieur):

$$
\frac{\mathrm{d} R}{\mathrm{~d} t}=K_{1}\left(\frac{\mathrm{d} E_{\mathrm{ox}}}{\mathrm{d} t}\right)^{0,5}-K_{2}
$$

où $K_{1}$ et $K_{2}$ sont des constantes empiriques. Les relations (1) et (2) sont valables aussi en atmosphère inerte où :

$$
\frac{\mathrm{d} E_{\mathrm{ox}}}{\mathrm{d} t}=0, \quad C_{\mathrm{i}}=C_{\mathrm{i}}^{*}, \quad C_{\mathrm{v}}=C_{\mathrm{v}}^{*}
$$

ce qui donne

$$
K_{2}=-\alpha\left[D_{\mathrm{i}}\left(C_{\mathrm{i}}^{*}-C_{\text {isf }}\right)+D_{\mathrm{v}}\left(C_{\mathrm{v}}^{*}-C_{\mathrm{vsf}}\right)\right]
$$

d'où la relation générale :

$$
\begin{aligned}
& D_{\mathrm{i}}\left(C_{\mathrm{i}}-C_{\mathrm{i}}^{*}\right)+D_{\mathrm{v}}\left(C_{\mathrm{v}}^{*}-C_{\mathrm{v}}\right)= \\
& =\frac{K_{1}}{K_{2}} \frac{\Delta G}{k T}\left(D_{\mathrm{i}} C_{\mathrm{i}}^{*}+D_{\mathrm{v}} C_{\mathrm{v}}^{*}\right)\left(\frac{\mathrm{d} E_{\mathrm{ox}}}{\mathrm{d} t}\right)^{0,5},
\end{aligned}
$$


sachant que $C_{\mathrm{i}, \mathrm{v}}^{*}$ est la concentration en I ou en $\mathrm{V}$ en ambiance inerte et que $\Delta G / k T$ est défini par ([14] et Réf. à l'intérieur) :

$$
\frac{C_{\text {isf }}}{C_{\mathrm{i}}^{*}}=\exp \left(\frac{\Delta G}{k T}\right)=\frac{C_{\mathrm{v}}^{*}}{C_{\mathrm{vsf}}} \simeq 1+\frac{\Delta G}{k T} .
$$

Si on suppose de plus que la réaction de Frenkel, $\mathrm{V}+\mathrm{I} \rightleftarrows$ Si substitutionnel, est à l'équilibre en tout point, on a :

$$
C_{\mathrm{v}}=\frac{C_{\mathrm{v}}^{*} C_{\mathrm{i}}^{*}}{C_{\mathrm{i}}}
$$

(3) devient alors :

$$
\begin{aligned}
\frac{C_{\mathrm{i}}}{C_{\mathrm{i}}^{*}}-\frac{D_{\mathrm{v}} C_{\mathrm{v}}^{*}}{D_{\mathrm{i}} C_{\mathrm{i}}^{*}} \frac{C_{\mathrm{i}}^{*}}{C_{\mathrm{i}}}= & \frac{K_{1}}{K_{2}} \frac{\Delta G}{k T}\left(1+\frac{D_{\mathrm{v}} C_{\mathrm{v}}^{*}}{D_{\mathrm{i}} C_{\mathrm{i}}^{*}}\right) \times \\
& \times\left(\frac{\mathrm{d} E_{\mathrm{ox}}}{\mathrm{d} t}\right)^{0,5}+1-\frac{D_{\mathrm{v}} C_{\mathrm{v}}^{*}}{D_{\mathrm{i}} C_{\mathrm{i}}^{*}} .
\end{aligned}
$$

On voit donc que, pour retrouver l'expression prise généralement pour $C_{\mathrm{i}} / C_{\mathrm{i}}^{*}$ qui est du type :

$$
\frac{C_{\mathrm{i}}}{C_{\mathrm{i}}^{*}}-1=K\left(\frac{\mathrm{d} E_{\mathrm{ox}}}{\mathrm{d} t}\right)^{0,5}
$$

il faut supposer que $D_{\mathrm{v}} C_{\mathrm{v}}^{*} \ll D_{\mathrm{i}} C_{\mathrm{i}}^{*}$, par suite dans la gamme de température considérée, l'auto-diffusion du silicium se ferait par phénomène interstitiel (nous reviendrons plus loin sur cette hypothèse). Faisant cette hypothèse, et prenant des temps d'oxydation suffisamment grands pour qu'on puisse ne prendre que le seul terme parabolique de la croissance d'oxyde, on a :

$$
\frac{C_{\mathrm{i}}}{C_{\mathrm{i}}^{*}}=K_{\mathrm{inj}} t^{-\frac{1}{4}}+1
$$

3.2 EQUATIONS DE DIFFUSION DES AUTO-INTERSTITIELS. - On part des équations classiques suivantes :

$$
\begin{aligned}
& \frac{\delta C_{\mathrm{i}}}{\delta t}=D_{\mathrm{i}} \frac{\delta^{2} C_{\mathrm{i}}}{\delta x^{2}}+k\left(C_{\mathrm{i}}^{*} C_{\mathrm{v}}^{*}-C_{\mathrm{i}} C_{\mathrm{v}}\right) \\
& \frac{\delta C_{\mathrm{v}}}{\delta t}=D_{\mathrm{v}} \frac{\delta^{2} C_{\mathrm{v}}}{\delta x^{2}}+k\left(C_{\mathrm{i}}^{*} C_{\mathrm{v}}^{*}-C_{\mathrm{i}} C_{\mathrm{v}}\right)
\end{aligned}
$$

(8a)-(8b) donne :

$$
\frac{\delta C_{\mathrm{i}}}{\delta t}-\frac{\delta C_{\mathrm{v}}}{\delta t}=D_{\mathrm{i}} \frac{\delta^{2} C_{\mathrm{i}}}{\delta x^{2}}-D_{\mathrm{v}} \frac{\delta^{2} C_{\mathrm{v}}}{\delta x^{2}} .
$$

On a vu que les populations relatives de I et de $\mathrm{V}$ étaient gouvernées par la réaction de Frenkel. Etant donné que l'équation (7b) donne, avec les temps de l'expérience considérée, des variations lentes de $C_{\mathrm{i}} / C_{\mathrm{i}}^{*}$, on va considérer dans l'équation $(8 \mathrm{c})$, contrairement à $[10,11]$, que la réaction de Frenkel est pratiquement à l'équilibre : $C_{\mathrm{i}}^{*} C_{\mathrm{v}}^{*} \# C_{\mathrm{i}} C_{\mathrm{v}}(5)$. Remarquons que ceci ne suppose pas le terme de recombinaison dans les équations (8a) et (8b) négligeable devant le terme de diffusion. En éliminant $C_{\mathrm{v}}$ entre (5) et (8c), on obtient alors l'équation de diffusion réduite (voir annexe) :

$$
\begin{aligned}
& \frac{\delta w}{\delta t}=\left(D_{\mathrm{i}}+\frac{D_{\mathrm{v}}-D_{\mathrm{i}}}{2 w^{2}+1+\frac{D_{\mathrm{v}}}{D_{\mathrm{i}}}+2 w \sqrt{w^{2}+\frac{D_{\mathrm{v}}}{D_{\mathrm{i}}}}}\right) \times \\
& \times \frac{\delta^{2} w}{\delta x^{2}}
\end{aligned}
$$

avec $w$ variable réduite :

$$
w=\frac{1}{2} \sqrt{\frac{C_{\mathrm{i}}^{*}}{C_{\mathrm{v}}^{*}}}\left(\frac{C_{\mathrm{i}}}{C_{\mathrm{i}}^{*}}-\frac{D_{\mathrm{v}} C_{\mathrm{v}}^{*}}{D_{\mathrm{i}} C_{\mathrm{i}}^{*}} \frac{C_{\mathrm{i}}^{*}}{C_{\mathrm{i}}}\right) .
$$

Dans cette variable réduite, on retrouve à un facteur près la grandeur donnée par l'équation (6) de croissance des fautes d'empilements. A l'hypothèse précédente, $D_{\mathrm{i}} C_{\mathrm{i}}^{*} \gg D_{\mathrm{v}} C_{\mathrm{v}}^{*}$, que nous avons faite pour obtenir l'équation (7a), nous rajoutons l'hypothèse que $D_{\mathrm{v}}$ est au moins égale à $D_{\mathrm{i}}$, ce qui entraîne que $C_{\mathrm{i}}^{*} \gg C_{\mathrm{v}}^{*}$ et débouche sur (voir annexe) :

$$
\frac{\delta C_{\mathrm{i}}}{\delta t}=D_{\mathrm{i}} \frac{\delta^{2} C_{\mathrm{i}}}{\delta x^{2}}
$$

qui est la même équation qu'en diffusion libre. $\mathrm{Ce}$ résultat n'est pas surprenant, puisque le seul terme de couplage qui est dû à la recombinaison affecte très peu la population majoritaire, à savoir ici $C_{\mathrm{i}}$. Nous voyons donc que l'on peut très bien supposer que la réaction de Frenkel a lieu et avoir une équation de diffusion simple moyennant une ou deux hypothèses déjà évoquées dans d'autres cas. Ces hypothèses entraînent que les lacunes ont une influence négligeable sur les fautes d'empilement et sur les auto-interstitiels. Par contre, les lacunes qui sont fortement minoritaires sont gouvernées essentiellement par la population des auto-interstitiels suivant l'équation (5). Nous raisonnons désormais avec cette équation pour alléger les calculs, mais on pourrait très bien simuler l'équation (9) directement. Nous partons de cette équation de base pour décrire le processus de diffusion dans le volume de silicium FZ (pas de recombinaison sur des centres fixes). Dans le silicium $\mathrm{CZ}$, on rajoute une recombinaison en volume du $1^{\text {er }}$ ordre des autointerstitiels sur les précipités d'oxygène avec une constante de temps $\tau$ dans l'équation (8a). L'équation (11) devient alors, avec les approximations signalées plus haut :

$$
\frac{\delta C_{\mathrm{i}}}{\delta t}=D_{\mathrm{i}} \frac{\delta^{2} C_{\mathrm{i}}}{\delta x^{2}}-\left(\frac{C_{\mathrm{i}}-C_{\mathrm{i}}^{*}}{\tau}\right) .
$$

Ceci n'est possible que si l'on suppose que les précipités d'oxygène n'influent pas directement sur les lacunes, afin de pouvoir garder l'équation (8b) intacte. 
Etant donné le manque de données sur le comportement réel des précipités d'oxygène, nous nous contenterons de cette hypothèse très simple que nous ne discuterons pas par la suite.

Sur la surface d'oxyde passivée nous prenons aussi une réaction du $1^{\text {er }}$ ordre, ce qui donne :

$$
\left.D_{\mathrm{i}} \frac{\delta C_{\mathrm{i}}}{\delta x}\right|_{\mathrm{SiO}_{2}+\mathrm{Si}_{3} \mathrm{~N}_{4}}=-k_{\mathrm{s}}\left(C_{\mathrm{i}}-C_{\mathrm{i}}^{*}\right) .
$$

Sur la surface de $\mathrm{Si}_{3} \mathrm{~N}_{4}$ seul, on suppose qu'il n'y a pas de recombinaison:

$$
\left.D_{\mathrm{i}} \frac{\delta C_{\mathrm{i}}}{\delta x}\right|_{\mathrm{Si}_{3} \mathrm{~N}_{4}}=0
$$

3.3 Diffusion DE L'IMPURETÉ Sb. - Nous supposons enfin l'effet des auto-interstitiels sur la diffusivité de l'impureté Sb comme connu $[5,11]$ empiriquement :

$$
\frac{D_{\mathrm{Sb}}}{D_{\mathrm{Sb}}^{*}}=f_{\mathrm{i}} \frac{C_{\mathrm{i}}}{C_{\mathrm{i}}^{*}}+\left(1-f_{\mathrm{i}}\right) \frac{C_{\mathrm{i}}^{*}}{C_{\mathrm{i}}}
$$

$f_{\mathrm{i}}=0,02$ pour $\mathrm{Sb}$ à $1100^{\circ} \mathrm{C}$

où $\frac{D_{\mathrm{Sb}}}{D_{\mathrm{Sb}}^{*}}$

est le rapport de la diffusivité de $\mathrm{Sb}$ en atmosphère oxydante à celle en atmosphère inerte. Cette quantité est déterminée expérimentalement dans l'expérience de Mizuo et Higuchi [5] par la mesure, d'une part de la profondeur de jonction $\left(x_{\mathrm{jbo}}\right)$ dans la zone faisant face à l'oxydation face arrière, d'autre part de la profondeur de jonction $\left(x_{\mathrm{jbn}}\right)$ dans une zone que l'on suppose assez éloignée de l'oxydation pour que $\mathrm{Sb}$ diffuse comme en ambiance inerte. Le rapport de ces 2 valeurs est supposé être relié au rapport des diffusivités par :

$$
\frac{x_{\mathrm{jbo}}}{x_{\mathrm{jbn}}}=\left(\frac{D_{\mathrm{Sb}}}{D_{\mathrm{Sb}}^{*}}\right)^{0,5} \text {. }
$$

Il est clair qu'en conjuguant les équations (15) et (16), on obtient le rapport $\frac{x_{\mathrm{jbo}}}{x_{\mathrm{jbn}}}$ en fonction de $\frac{C_{\mathrm{i}}}{C_{\mathrm{i}}^{*}}$.

Il est à noter que nous avons choisi de traiter l'impureté Sb plutôt que le bore ou le phosphore en raison du meilleur accord qui existe dans la littérature sur la valeur de $f_{\mathrm{i}}$ à $11^{\circ} \mathrm{C}$ pour l'antimoine. On peut voir aussi que l'équation (15) est moins sensible à l'erreur sur $f_{\mathrm{i}}$ si celui-ci est petit.

3.4 Démarche DE LA Simulation. - Nous avons ajusté le rapport $x_{\mathrm{jbo}} / x_{\mathrm{jbn}}$ (mesuré expérimentalement), par l'intermédiaire de $C_{\mathrm{i}} / C_{\mathrm{i}}^{*}$ noté par la suite $S_{\mathrm{i}}$ et calculé sur la face avant avec les équations et les conventions suivantes :

- face avant : $x=L$ ( $L$ allant de 100 à $400 \mu \mathrm{m})$,

- face arrière : $x=0$ (voir Fig. 3).

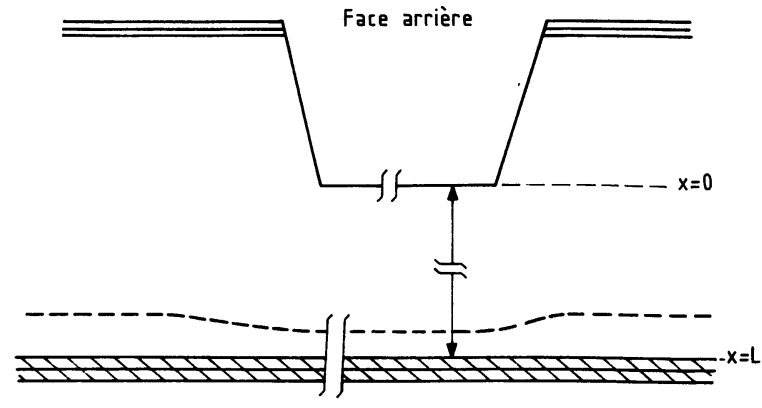

Fig. 3. - Conventions prises pour les bornes.

[Conventions for the boundaries.]

L'équation (7b) nous donne :

$$
S_{\mathrm{i}}(x=0, t)=K_{\mathrm{inj}} t^{-\frac{1}{4}}+1,
$$

pour le cas d'un substrat FZ (recombinaison des I avec les $\mathrm{V}$ seulement), l'équation (11) donne :

$$
\frac{\delta S_{\mathrm{i}}}{\delta t}(x, t)=D_{\mathrm{i}} \frac{\delta^{2} S_{\mathrm{i}}}{\delta x^{2}}(x, t)
$$

pour le cas d'un substrat CZ, l'équation (12) donne :

$$
\frac{\delta S_{\mathrm{i}}}{\delta t}(x, t)=D_{\mathrm{i}} \frac{\delta^{2} S_{\mathrm{i}}}{\delta x^{2}}(x, t)-\left(\frac{S_{\mathrm{i}}(x, t)-1}{\tau}\right)
$$

pour le cas où la face avant comporte une couche de $\mathrm{Si}_{3} \mathrm{~N}_{4}$ seule, l'équation (14) donne :

$$
D_{\mathrm{i}} \frac{\delta S_{\mathrm{i}}}{\delta x}(x=L, t)=0,
$$

pour le cas où la face avant comporte une couche de $\mathrm{SiO}_{2}$ plus une couche de $\mathrm{Si}_{3} \mathrm{~N}_{4}$, l'équation (13) donne :

$$
D_{\mathrm{i}} \frac{\delta S_{\mathrm{i}}}{\delta t}(x=L, t)=-k_{\mathrm{s}}\left(S_{\mathrm{i}}(x=L, t)-1\right) .
$$

Nous avons considéré que les paramètres ajustables étaient dans un premier temps $D_{\mathrm{i}}$ et $K_{\text {inj }}$ (simulation du cas substrat $\mathrm{FZ}+\mathrm{Si}_{3} \mathrm{~N}_{4}$ seul avec les équations (17), (18), (20)). Puis, nous avons pris le meilleur ajustement de ces 2 paramètres pour obtenir $\tau$ et $k_{\mathrm{s}}$ (simulation des autres cas). Il faut remarquer qu'il existe déjà des valeurs de $K_{\text {inj }}$ dans la littérature $[13,15,16]$, et qu'on aurait pu les utiliser. Néanmoins ces valeurs sont déterminées à partir d'expériences en face avant et on sait que la cinétique de production d'autointerstitiels dépend beaucoup de l'état de surface. La surface oxydée ici est le substrat du silicium et de plus elle a subi une attaque chimique violente. Nous avons donc pensé a priori que la production des autointerstitiels pouvait ne pas être la même qu'en face avant. 


\section{Résultats de la simulation et commentaires.}

4.1 $D_{\mathrm{i}}$ ET $K_{\text {in j }}$ - La simulation faite sur le premier cas (substrat $\mathrm{FZ}$ et $\mathrm{Si}_{3} \mathrm{~N}_{4}$ seul) donne un bon ajustement des valeurs empiriques ainsi qu'on peut le voir sur la figure 4, cet ajustement a été obtenu avec :

$$
K_{\mathrm{inj}}=4,2(\min )^{1 / 4} \text { et } D_{\mathrm{i}}=13 \mu \mathrm{m}^{2} \cdot \min ^{-1},
$$

(valeurs ajustées à $5 \%$ près). On peut déjà discuter de la cohérence de ces résultats : la valeur de $K_{\text {inj }}$ est remarquablement proche des valeurs données dans la littérature. En effet, on trouve selon les auteurs :

$$
\begin{aligned}
K_{\text {inj }} & =4,68(\min )^{1 / 4} \\
& =4,19(\min )^{1 / 4}, \\
& =3,33(\min )^{1 / 4}
\end{aligned}
$$

(resp. $[13,15,16])$.

Par contre, la valeur de $D_{i}$ est beaucoup plus petite que celle qu'on trouve généralement dans la littérature. La seule valeur concordante est celle trouvée par
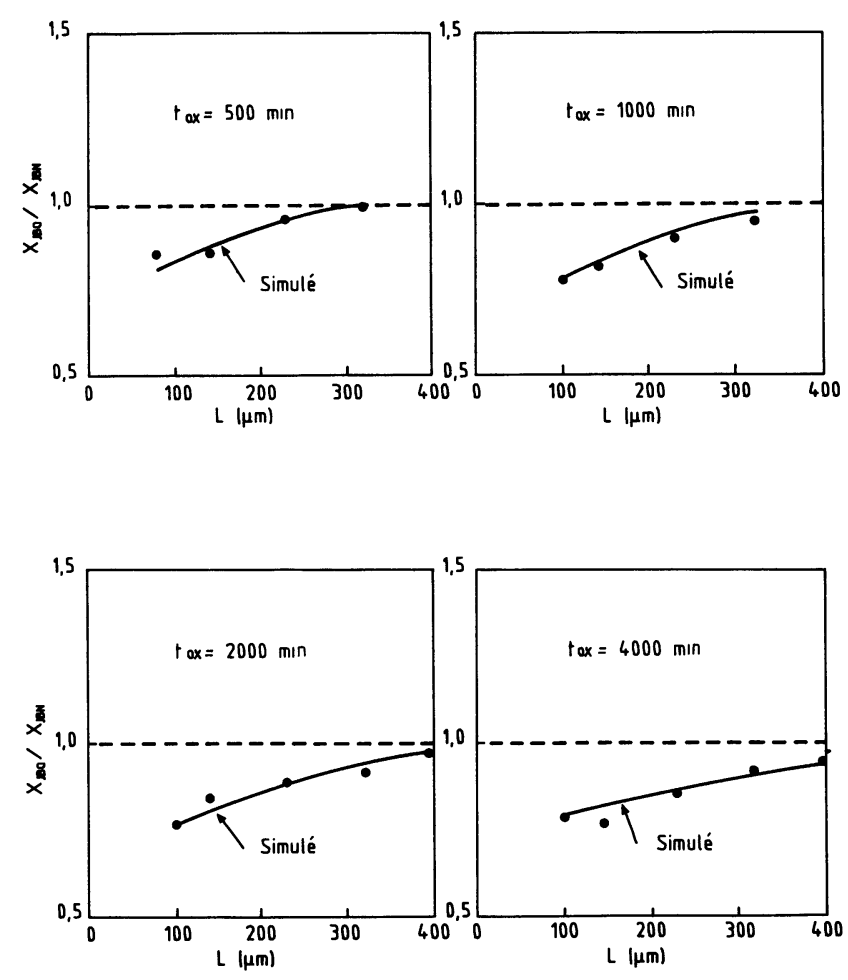

Fig. 4. $-x_{\mathrm{jbo}} / x_{\mathrm{jbn}}$ (profondeur de jonction dans la zone oxydée par rapport à la profondeur de jonction dans la zone non oxydée) en fonction de $L$, épaisseur résiduelle de la plaquette pour différents temps, $t_{\mathrm{ox}}$, d'oxydation. Résultats expérimentaux et simulés, dans le cas silicium $\mathrm{FZ}$ et $\mathrm{Si}_{3} \mathrm{~N}_{4}$ seul.

$\left[x_{\mathrm{jbo}} / x_{\mathrm{jbn}}\right.$ (junction depth in oxidized area versus junction depth in non-oxidized one) function of $L$, residual width of the wafer for different times, $t_{\mathrm{ox}}$, of oxidation. Experimental and simulated results, in the case silicon $\mathrm{FZ}$ and $\mathrm{Si}_{3} \mathrm{~N}_{4}$ alone.]
Taniguchi et al. [8], mais l'expérience d'où il tire sa valeur n'est pas cohérente avec l'expérience traitée ici, [5] (substrat CZ sans recombinaison, etc...). Nous reviendrons plus loin $(\$ 4.3)$ sur cette contradiction.

$4.2 k_{\mathrm{s}}$ ET $\tau$. - Avec les deux valeurs trouvées précédemment, on a simulé les cas substrat $\mathrm{CZ}$ ou surface passivée $\mathrm{Si}_{3} \mathrm{~N}_{4}+\mathrm{SiO}_{2}$ et on a trouvé :

$k_{\mathrm{s}}=1,5 \mu \mathrm{m} \cdot \mathrm{min}^{-1}$ et $\tau=105 \mathrm{~min}$ ce qui donne :

$L_{\mathrm{s}}=\frac{D_{\mathrm{i}}}{k_{\mathrm{s}}}=8 \mu \mathrm{m} \quad$ et $\quad L_{\mathrm{v}}=\sqrt{D_{\mathrm{i}} \tau}=36 \mu \mathrm{m}$.

Il est à noter, qu'étant donné le manque de résultats significatifs dans l'expérience, ces résultats sont moins précis, l'erreur étant alors de l'ordre de $40 \%$ sur $k_{\mathrm{s}}$ et $\tau$. Cependant, il est facile de constater que :

a) l'ordre de grandeur de la longueur de recombinaison en surface, $L_{\mathrm{s}}$, se retrouve dans les expériences 2-D : [7, 17, 18]. L'expérience de Taniguchi et al. [8] par contre donne $k_{\mathrm{s}} \# 4 \times 10^{-5} \mu \mathrm{m} / \mathrm{min}$, soit $L_{\mathrm{s}}=$ $25 \mathrm{~cm}$ ! ce qui est infiniment plus grand que tout ce qui a été constaté en effet $2-\mathrm{D}\left(L_{\mathrm{s}}=25 \mathrm{~cm}\right.$ signifie par exemple que l'ordre de grandeur de la zone de transition de la figure 1 est de quelques centimètres). De ce fait, en rappelant ce qui a été dit en introduction, la valeur que nous trouvons peut être considérée, malgré sa faible précision, comme la plus fiable parmi tout ce qui a été publié;

b) l'ordre de grandeur de la longueur de recombinaison en volume, $L_{v}$, correspond aussi à ce qui a été trouvé en $2-\mathrm{D}:[6,19]$. On peut faire la même conclusion sur la valeur de $\tau$ par rapport aux valeurs trouvées par exemple pour des couches enterrées [6, 19].

4.3 COMParaison AVEC LES RÉSUltats DE L'EXPÉRIENCE DE TANIGUCHI et al. - L'expérience de Taniguchi et al. [8] est aussi une expérience d'oxydation en face arrière, mais les auteurs ont étudié son effet sur des fautes d'empilements situées en face avant sous une couche de $\mathrm{SiO}_{2}$ passivée. Ils ont constaté qu'il n'y avait pas de différence de comportement entre substrat FZ et $\mathrm{CZ}$ (donc pas de recombinaison en volume avec une constante de temps $\tau$ ) et que la recombinaison sur la surface de $\mathrm{SiO}_{2}$ était très faible $\left(k_{\mathrm{s}}=4 \times 10^{-5} \mu \mathrm{m} / \mathrm{min}\right.$ à $\left.1100^{\circ} \mathrm{C}\right)$. Ainsi, cette expérience est en contradiction avec les résultats $2-\mathrm{D}$ et aussi avec la même expérience faite par Mizuo et Higuchi [20]. En effet, ceux-ci trouvent une bonne corrélation entre la cinétique de croissance des fautes d'empilement et la diffusivité de la couche d'impuretés en face avant. Enfin, Taniguchi et al. ont eux aussi [21] simulé l'expérience [5], dans le cas "substrat $\mathrm{FZ}+\mathrm{Si}_{3} \mathrm{~N}_{4}$ " en prenant le modèle élaboré pour le cas "substrat $\mathrm{CZ}+\mathrm{SiO}_{2}$ " et la même valeur de $D_{i}$. Le fait que cette simulation soit peu cohérente nous fait préférer les valeurs trouvées par notre simulation. A notre avis, le désaccord peut venir de ce que l'attaque chimique d'amincissement utilisée par Taniguchi et al. a pu changer l'état 
de surface et donc le taux de création des auto-interstitiels.

4.4 Comparaison AVEC Les impuretés B ET P. Mizuo et Higuchi ayant procédé aux mêmes expériences mais avec du bore ou du phosphore en face avant $[5,22]$, nous avons refait les mêmes simulations avec les valeurs de $K_{\mathrm{inj}}, D_{\mathrm{i}}, k_{\mathrm{s}}, \tau$. Le résultat est en bon accord avec les données expérimentales, mais en prenant $f_{\mathbf{i}} \sim 0,9$; c'est-à-dire qu'il faut admettre que la diffusion de ces impuretés est presque uniquement " interstitiellement assistée "; alors que, par exemple, la diffusion extrinsèque du bore s'explique très bien avec une diffusion uniquement lacunaire [23]. Nous reviendrons plus loin sur ce problème.

\subsection{DISCUSSION SUR L'HYPOTHÈSE DE RECOMBINAISON A LA SURFACE.}

4.5.1 Sur $\mathrm{SiO}_{2}$ passivé. - Mizuo et Higuchi ont invoqué une réaction sur $\mathrm{SiO}_{2}$ passivé du type :

$$
\mathrm{SiO}_{2}+\mathrm{Si}_{\mathrm{I}} \rightarrow 2 \mathrm{SiO}
$$

qui est à rapprocher de la réaction de recombinaison dans le modèle proposé par Lin et al. [24] :

$$
\mathrm{Si}_{\mathrm{I}}+\frac{1}{2} \mathrm{O}_{2} \rightarrow \mathrm{SiO}
$$

ou, plus généralement :

$$
\mathrm{Si}_{m} \mathrm{O}_{n}+\mathrm{Si}_{\mathrm{I}} \rightarrow \mathrm{Si}_{m+1} \mathrm{O}_{n}
$$

s'il y a des liaisons partagées. Cela signifie d'abord que sous tout oxyde thermique, il existe une couche de transition du type $\mathrm{SiO}_{x}$. Ensuite, on peut se demander si la réaction de recombinaison ne sature pas du fait d'une trop grande quantité d'interstitiels fixés à l'interface, en effet on avait (Eq. (13)) :

$$
\left.D_{\mathrm{i}} \frac{\delta C_{\mathrm{i}}}{\delta x}\right|_{x=L}=k_{\mathrm{s}}\left(C_{\mathrm{i}}(L)-C_{\mathrm{i}}^{*}\right)
$$

or au temps $t$, l'interface a absorbé :

$$
\left.\int_{0}^{t} D_{\mathrm{i}} \frac{\delta C_{\mathrm{i}}}{\delta x}\right|_{x=L} \mathrm{~d} t \text { atomes par } \mathrm{cm}^{2}
$$

soit

$$
\int_{0}^{t} k_{\mathrm{s}}\left(C_{\mathrm{i}}(L)-C_{\mathrm{i}}^{*}\right) \mathrm{d} t
$$

soit

$$
N=k_{\mathrm{s}} C_{\mathrm{i}}^{*} t\left(\left\langle\frac{C_{\mathrm{i}}(L)}{C_{\mathrm{i}}^{*}}\right\rangle_{t}-1\right) .
$$

Notre programme nous donnant la valeur relative moyenne de $C_{\mathrm{i}}$, il nous est facile de déterminer en fonction de $C_{\mathrm{i}}^{*}$ le nombre d'atomes absorbés par unité de surface, par exemple pour $t=1000 \min$ et $L=$
$100 \mu \mathrm{m}$, on obtient :

$$
\begin{array}{lll}
C_{\mathrm{i}}^{*}=10^{14} \mathrm{~cm}^{-3} & \text { donne } & N=10^{12} \text { at. } \mathrm{cm}^{-2} \\
C_{\mathrm{i}}^{*}=10^{17} \mathrm{~cm}^{-3} & \text { donne } & N=10^{15} \text { at. } \mathrm{cm}^{-2},
\end{array}
$$

à comparer avec la densité surfacique du Si qui est $1,5 \times 10^{15}$ at $\mathrm{cm}^{-2}$. On voit alors que selon la valeur de $C_{i}^{*}$ qu'on prend, on peut avoir saturation de la couche de $\mathrm{Si}$ à l'interface $\mathrm{Si}_{-} \mathrm{SiO}_{2}$. Or, il ne faut pas oublier qu'une hypothèse de notre modélisation était que $C_{\mathrm{i}}^{*} \gg C_{\mathrm{v}}^{*}$, donc en concentration relativement importante, d'où une objection possible à notre travail. Dans le travail de Shin et Kim [4], qui, avec un modèle similaire au nôtre, donne une $L_{\mathrm{s}}$ du même ordre, on peut voir que $k_{\mathrm{s}}$ est $10^{3}$ fois plus grand (ainsi que $D_{\mathrm{i}}$ ), d'où une saturation quasi certaine de la réaction de recombinaison. Il est donc justifié de s'interroger sur la validité physique des modèles invoquant cette recombinaison

4.5.2 Sur la surface de $\mathrm{Si}_{3} \mathrm{~N}_{4}$ - - Nous avons supposé qu'il n'y avait pas recombinaison à la surface $\mathrm{du} \mathrm{Si}_{3} \mathrm{~N}_{4}$. En fait il suffit que la longueur de recombinaison soit grande devant l'épaisseur résiduelle de la plaquette, ce qui est plausible au vu d'une expérience 2-D [25]. Mais l'expérience de la référence [25] nous apporte une contradiction de taille : l'effet de l'oxydation sur les impuretés situées sous $\mathrm{Si}_{3} \mathrm{~N}_{4}$ est moins fort en substrat $\mathrm{FZ}$ qu'en substrat $\mathrm{CZ}$, il est même moins fort que sous $\mathrm{SiO}_{2}+\mathrm{Si}_{3} \mathrm{~N}_{4}$ dans certains cas. C'est exactement le contraire de ce qui est constaté dans l'expérience simulée ici. Cela ne nous gène pas dans la mesure où nous avons traité un effet différentiel (mesure de $x_{\mathrm{jbo}} / x_{\mathrm{jbn}}$ ) mais notre modèle ne peut pas décrire correctement l'effet du nitrure seul. Cette contradiction ne peut être levée que par une meilleure connaissance des propriétés physiques d'un dépôt de nitrure sur silicium en présence d'oxygène et notamment en ce qui concerne l'influence des contraintes provoquées par les différences de maille entre $\mathrm{Si}_{3} \mathrm{~N}_{4}$ et $\mathrm{Si}$.

\section{Commentaires sur les hypothèses et résultats phy- siques.}

5.1 $D_{\mathrm{i} .}$ - Comme Goesele [26], nous trouvons une valeur de $D_{\mathrm{i}}$ trop faible. Goesele [26] avance que l'on a $C_{\mathrm{v}}^{*} \gg C_{\mathrm{i}}^{*}$ (toujours avec $D_{\mathrm{v}} C_{\mathrm{v}}^{*} \ll D_{\mathrm{i}} C_{\mathrm{i}}^{*}$ ) à l'opposé de notre hypothèse et qu'alors la diffusivité mesurée est :

$$
D_{\text {eff }}=\frac{D_{\mathrm{i}} C_{\mathrm{i}}^{*}}{C_{\mathrm{v}}^{*}} \text { on a ainsi : } D_{\text {eff }} \ll D_{\mathrm{i}} .
$$

Mais cela impliquerait que :

$$
D_{\mathrm{v}} \ll D_{\mathrm{i}} \quad \text { et } \quad D_{\mathrm{v}} \ll 10 \mu \mathrm{m}^{2} \cdot \min ^{-1} \text { à } 1100^{\circ} \mathrm{C}
$$

ce qui est peu réaliste [27,28]. De plus, ceci ne peut être expliqué par le fait que la réaction de Frenkel n'est pas assez rapide pour pouvoir supposer $C_{\mathrm{i}} C_{\mathrm{v}} \# C_{\mathrm{i}}^{*} C_{\mathrm{v}}^{*}$. 
En effet, si la réaction de Frenkel n'est pas à l'équilibre, ceci conduit à un effet des lacunes et interstitiels sur la diffusivité des impuretés plus important pour une même diffusivité $D_{i}$. Donc, pour obtenir le même rapport $D_{\text {imp }} / D_{\text {imp* }}$, il faut avoir $D_{\mathrm{i}}$ plus petit (ou $D_{\mathrm{i}} C_{\mathrm{i}}^{*} / C_{\mathrm{v}}^{*}$ plus petit).

5.2 AUTO-DIFFUSION DANS LE SILICIUM ET PHÉNOMÈNES DÉRIVÉS. - Il nous faut, pour avoir un départ cohérent avec les équations admises (Eq. (7a)) pour la croissance des fautes d'empilements, admettre que l'auto-diffusion du silicium se fait, au moins dans la gamme de température $900^{\circ} \mathrm{C}-1100^{\circ} \mathrm{C}$, uniquement par phénomène interstitiel, hypothèse mise en question par Bourgoin [29] et Van Vechten [30], au vu d'expériences indépendantes sur l'auto-diffusion et l'existence de défauts ponctuels. Il nous faut aussi admettre que la plupart des impuretés diffusent interstitiellement aux environs de $1100^{\circ} \mathrm{C}$, ce qui est aussi remis en cause par Bourgoin ([27, 29]) et Van Vechten ([28]).

Par ailleurs, le travail de Mathiot et Pfister [31] montre qu'en supposant une diffusion essentiellement lacunaire pour les impuretés, on trouve une valeur de $K_{\text {inj }}$ beaucoup plus grande que la valeur expérimentale déterminée directement à partir des fautes d'empilement induites par oxydation. Nous pensons, au vu de tout ce qui précède, qu'il faut peut-être dissocier les comportements des fautes d'empilement et des impuretés sous oxydation, si ce n'est qu'en modifiant l'équation (3) de croissance des fautes d'empilement. Ceci nécessite bien sûr d'être exploré par l'intermédiaire de nouvelles expériences.

Ceci est conforté par les considérations sur les contraintes induites par l'oxydation thermique (travaux de Tan et Goesele [32], Eernisse [33], Brotherton et al. [34]), confirmées par les résultats de Fargeix et Ghibaudo [35], qui montrent que le silicium est en tension sous l'oxyde. Il y a donc a priori une tendance à créer des lacunes ou des défauts de type lacunaire plutôt que des interstitiels ou des défauts de type interstitiel. Van Vechten [28] a proposé que des " vides » soient présents en même temps que les fautes d'empilements interstitielles mais qu'ils sont peu observables par microscopie ou qu'ils recristallisent très rapidement quand on descend en température. Il a aussi proposé plus récemment l'existence d'une lacune "étendue amorphe » c'est-à-dire l'existence d'une zone à $N$ mailles sous-jacentes où la densité est moindre [30]. L'existence de vides est aussi invoquée par Bourgoin [29] pour expliquer la différence entre l'énergie de formation du défaut déterminée par les méthodes de trempe et l'énergie de formation déterminée par l'auto-diffusion elle-même. Ces hypothèses sont séduisantes pour expliquer les observations faites pour l'oxydation mais demandent d'être reliées quantitativement aux contraintes dans le silicium et sans doute une révision complète du modèle physique.

Revue de PHysique appliquf́e. - T. 20, № 7, JULlett 1985

\section{Conclusion.}

Nous avons élaboré un modèle simple pour la distribution des auto-interstitiels (et des lacunes) en atmosphère oxydante. Ce modèle donne la diffusivité $D_{i}$ des auto-interstitiels, la constante de recombinaison en surface $k_{\mathrm{s}}$ sur du $\mathrm{SiO}_{2}$ passivé, la constante de recombinaison $\tau$ en volume dans du substrat $\mathrm{CZ}$. Ainsi le programme de simulation qui repose sur ce modèle peut compléter un programme de simulation bidimensionnel, chose qui répondrait aux nouveaux besoins créés par l'évolution des processus technologiques vers des dimensions submicroniques.

Ayant donc montré que le modèle que nous avons déterminé peut servir à décrire de façon satisfaisante les phénomènes de diffusion sous oxydation en 2 dimensions dans la structure LOCOS (Fig. 1), il peut donc $a$ fortiori aider à décrire la cinétique de l'oxydation dans la zone dite du " bec d'oiseau » (zone de transition de l'oxyde dans la Fig. 1). Dans cette optique, des expériences similaires à des températures différentes seraient nécessaires pour avoir un modèle complet et opérationnel.

Par contre, il faut considérer que la physique de ce modèle reste pour le moment en question. S'il est toujours possible d'ajuster des résultats empiriques particuliers à une expérience en jouant sur un grand nombre de paramètres, un peu comme nous l'avons fait, il faut constater que certaines hypothèses physiques faites par nous-mêmes et d'autres auteurs débouchent sur des résultats contradictoires avec d'autres expériences. Il faut donc trouver des expériences indépendantes pour pouvoir infirmer ou confirmer les théories jusqu'alors proposées. Il est ainsi nécessaire d'étudier l'effet de contraintes de faible intensité $\left(10^{7}-10^{8} \mathrm{dyn} / \mathrm{cm}^{2}\right)$ sur la diffusion des impuretés et sur les défauts macroscopiques.

\section{Annexe.}

On note : $P=C_{\mathrm{i}}^{*} C_{\mathrm{v}}^{*},(5)$ donne alors :

$$
\begin{aligned}
\frac{\delta C_{\mathrm{v}}}{\delta t} & =-\frac{P}{C_{\mathrm{i}}^{2}} \frac{\delta C_{\mathrm{i}}}{\delta t} \\
\frac{\delta^{2} C_{\mathrm{v}}}{\delta x^{2}} & =-\frac{P}{C_{\mathrm{i}}^{2}} \frac{\delta^{2} C_{\mathrm{i}}}{\delta x^{2}}+\frac{2 P}{C_{\mathrm{i}}^{3}}\left(\frac{\delta C_{\mathrm{i}}}{\delta x}\right)^{2}
\end{aligned}
$$

ce qui donne dans $(8 \mathrm{c})$ :

$$
\left(P+C_{\mathrm{i}}^{2}\right) \frac{\delta C_{\mathrm{i}}}{\delta t}=\left(D_{\mathrm{i}} C_{\mathrm{i}}^{2}+P D_{\mathrm{v}}\right) \frac{\delta^{2} C_{\mathrm{i}}}{\delta x^{2}}-\frac{2 P D_{\mathrm{v}}}{C_{\mathrm{i}}}\left(\frac{\delta C_{\mathrm{i}}}{\delta x}\right)^{2}
$$

on fait le changement de variable suivant : $C_{\mathrm{i}}=u \sqrt{P}$ ce qui donne :

$$
\left(u^{2}+1\right) \frac{\delta u}{\delta t}=\left(D_{\mathrm{i}} u^{2}+D_{\mathrm{v}}\right) \frac{\delta^{2} u}{\delta x^{2}}-\frac{2 D_{\mathrm{v}}}{u}\left(\frac{\delta u}{\delta x}\right)^{2}
$$


on fait le nouveau changement de variable :

$$
\begin{gathered}
w=\frac{1}{2}\left(u-\frac{D_{\mathrm{v}}}{D_{\mathrm{i}} u}\right) \\
\frac{\delta w}{\delta t}=\frac{1}{2} \frac{\delta u}{\delta t}\left(1+\frac{D_{\mathrm{v}}}{D_{\mathrm{i}} u^{2}}\right)=\frac{D_{\mathrm{i}} u^{2}+D_{\mathrm{v}}}{2 D_{\mathrm{i}} u^{2}} \frac{\delta u}{\delta t} \\
\frac{\delta^{2} w}{\delta x^{2}}=\frac{1}{2} \frac{\delta^{2} u}{\delta x^{2}}\left(1+\frac{D_{\mathrm{v}}}{D_{\mathrm{i}} u^{2}}\right)-\frac{D_{\mathrm{v}}}{D_{\mathrm{i}} u^{3}}\left(\frac{\delta u}{\delta x}\right)^{2}
\end{gathered}
$$

soit :

$$
\frac{\delta^{2} w}{\delta x^{2}}=\frac{1}{2 D_{\mathrm{i}} u^{2}}\left(\left(D_{\mathrm{i}} u^{2}+D_{\mathrm{v}}\right) \frac{\delta^{2} u}{\delta x^{2}}-\frac{2 D_{\mathrm{v}}}{u}\left(\frac{\delta u}{\delta x}\right)^{2}\right)
$$

l'équation de diffusion devient alors :

$$
\frac{\delta w}{\delta t}=\frac{D_{\mathrm{i}} u^{2}+D_{\mathrm{v}}}{u^{2}+1} \frac{\delta^{2} w}{\delta x^{2}}=\left(D_{\mathrm{i}}+\frac{D_{\mathrm{v}}-D_{\mathrm{i}}}{u^{2}+1}\right) \frac{\delta^{2} w}{\delta x^{2}}
$$

d'où, en exprimant $u$ par rapport à $w$ :

$$
\begin{aligned}
& \frac{\delta w}{\delta t}=\left(D_{\mathrm{i}}+\frac{D_{\mathrm{v}}-D_{\mathrm{i}}}{2 w^{2}+1+\frac{D_{\mathrm{v}}}{D_{\mathrm{i}}}+2 w \sqrt{w^{2}+\frac{D_{\mathrm{v}}}{D_{\mathrm{i}}}}}\right) \times \\
& \times \frac{\delta^{2} w}{\delta x^{2}}=D_{\mathrm{i}} F(w) \frac{\delta^{2} w}{\delta x^{2}}
\end{aligned}
$$

ceci avec :

$$
w=\frac{1}{2}\left(u-\frac{D_{\mathrm{v}}}{D_{\mathrm{i}} u}\right)=\frac{1}{2}\left(\frac{C_{\mathrm{i}}}{\sqrt{C_{\mathrm{i}}^{*} C_{\mathrm{v}}^{*}}}-\frac{D_{\mathrm{v}} \sqrt{C_{\mathrm{i}}^{*} C_{\mathrm{v}}^{*}}}{D_{\mathrm{i}} C_{\mathrm{i}}}\right),
$$

soit :

$$
w=\frac{1}{2} \sqrt{\frac{C_{\mathrm{i}}^{*}}{C_{\mathrm{v}}^{*}}}\left(\frac{C_{\mathrm{i}}}{C_{\mathrm{i}}^{*}}-\frac{D_{\mathrm{v}} C_{\mathrm{v}}^{*}}{D_{\mathrm{i}} C_{\mathrm{i}}^{*}} \frac{C_{\mathrm{i}}^{*}}{C_{\mathrm{i}}}\right) .
$$

Cas où $D_{\mathrm{i}} C_{\mathrm{i}}^{*} \gg D_{\mathrm{v}} C_{\mathrm{v}}^{*}$ et $C_{\mathrm{i}}^{*} \gg C_{\mathrm{v}}^{*}$ :

$$
w \# \frac{1}{2} \sqrt{\frac{C_{\mathrm{i}}^{*}}{C_{\mathrm{v}}^{*}}} \frac{C_{\mathrm{i}}}{C_{\mathrm{i}}^{*}}
$$

donc $w$ est proportionnel à $C_{\mathrm{i}}$

donc :

$$
w^{2}=\frac{1}{4} \frac{C_{\mathrm{i}}^{*}}{C_{\mathrm{v}}^{*}}\left(\frac{C_{\mathrm{i}}}{C_{\mathrm{i}}^{*}}\right)^{2} \gg 1 \text { et } \gg \frac{D_{\mathrm{v}}}{D_{\mathrm{i}}}
$$

$$
F(w) \# 1+\frac{D_{\mathrm{v}} / D_{\mathrm{i}}-1}{4 w^{2}} \# 1,
$$

l'équation de diffusion devient :

$$
\frac{\delta w}{\delta t}=D_{\mathrm{i}} \frac{\delta^{2} w}{\delta x^{2}} \text { soit } \frac{\delta C_{\mathrm{i}}}{\delta t}=D_{\mathrm{i}} \frac{\delta^{2} C_{\mathrm{i}}}{\delta x^{2}} .
$$

\section{Bibliographie}

[1] Chin, D., Kump, M. R., Lee, H. G. et Dutton, R. W., IEEE Trans. Electron Devices ED 29 (1982) 336.

[2] Guillemot, N., Chenevier, P., Deroux-Dauphin, P. et Gonchond, J. P., Revue Phys. Appl., à paraître.

[3] Collard, D., Lobet, M., Decarpigny, J. N., 14th European Solid State Device Research Conference, Lille, édité par Zimmermann, J. (1984), 178.

[4] SHIN, Y. S. et KIM, C. K., IEEE Trans. Electron Devices ED 20 (1982) 797.

[5] Mizuo, S. et Higuchi, H., J. Electrochem. Soc. 130 (1983) 1942.

[6] Taniguchi, K., Kurosawa, K. et Kashiwagi, M., J. Electrochem. Soc. 127 (1980) 2243.

[7] Lin, A. M., Dutton, R. W. et Antoniadis, D. A., Appl. Phys. Lett. 35 (1979) 799.

[8] Taniguchi, K., Antoniadis, D. A. et Matsushita, Y., Appl. Phys. Lett. 42 (1983) 961.

[9] Hu, S. M., J. Appl. Phys. 45 (1974) 1567.

[10] Mizuo, S. et Higuchi, H., Jpn. J. Appl. Phys. 20 (1981) 739.

[11] Antoniadis, D. A. et Moskowitz, I., Proceedings of the Satellite Symposium to ESSDERC' $82, \mathrm{Mu}-$ nich, édité par Sirti et Goorissen (Electrochem. Soc. Inc.) 1983, p. 1.

[12] Tan, T. Y. et Goesele, U., Appl. Phys. Lett. 40 (1982) 616.

[13] Antoniadis, D. A., Semiconductor Silicon 1981, édité par Huff, Kriegler et Takeishi (Electrochem. Soc. Inc.) 1981 , p. 947
[14] Goesele, U. et Frank, W., Defects in Semiconductors, vol. 2, édité par Narayan et Tan (North-Holland) 1981, p. 55.

[15] Tan, T. Y. et Ginsberg, B. J., Appl. Phys. Lett. 42 (1983) 448.

[16] Matsumoto, S., Ishikawa, Y. et Nirmi, T., J. Appl. Phys. 54 (1983) 5049.

[17] Mizuo, S. et Higuchi, H., Jpn. J. Appl. Phys. 21 (1982) 281

[18] Mizuo, S. et Higuchi, H., Jpn. J. Appl. Phys. 20 (1981) 791.

[19] Hill, C., Semiconductor Silicon 1981, édité par Huff, Kriegler et Takeishi (Electrochem. Soc. Inc.) 1981, p. 989.

[20] Mizuo, S. et Higuchi, H., Jpn. J. Appl. Phys. 21 (1982) 1547.

[21] Taniguchi, K. et Antoniadis, D. A., Extended Abst Spring Meeting San Francisco 1983, 83-1 (édité par Electrochem. Soc. Inc.) 1983, p. 438.

[22] Mizuo, S. et Higuchi, H., J. Electrochem. Soc. 129 (1982) 2292.

[23] Tsoukalas, D. et Chenevier, P., Extended Abst. Spring Meeting San Francisco 1983, 83-1 (édité par Electrochem. Soc. Inc.) 1983, p. 436.

[24] Lin, A. M., Dutton, R. W., Antoniadis, D. A. et Tiller, W. A., J. Electrochem. Soc. 128 (1981) 1121.

[25] Mizuo, S. et Higuchi, H., Jpn. J. Appl. Phys. 21 (1982) 281. 
[26] Goesele, U. et TAN, T. Y., Defects in Semiconductors II édité par Mahajan et Corbett (North-Holland) 1983, p. 45.

[27] Bourgoin, J. C., Phys. Rev. B 29 (1984) 4789.

[28] Van Vechten, J. C., Phys. Rev. B 17 (1978) 3197.

[29] Bourgorn, J. C., Proceedings of 13th International Conference on Defects in Semiconductors, Coronado (édité par J. Electron. Mat.) 1984, à paraître.

[30] Van Vechten, J. C., idem.

[31] Mathiot, D. et Pfister, J. C., J. Appl. Phys. 55 (1984) 3519.
[32] Tan, T. Y. et Goesele, U., Appl. Phys. Lett. 39 (1981) 86.

[33] Eernisse, E. P., Appl. Phys. Lett. 30 (1977) 290 et 35 (1979) 8.

[34] Brotherton, S. D., Read, T. G., Lamb, D. R. et WilloughBY, F. W., Solid State Electron. 16 (1973) 1367.

[35] Fargeix, A. et Ghibaudo, G., J. Appl. Phys. 54 (1983) 7153. 